\title{
A Branch-and-Cut Algorithm for the Alternative Fuel Refueling Station Location Problem with Routing
}

\author{
Okan Arslan, ${ }^{\text {a }}$ Oya Ekin Karaşan, ${ }^{b}$ A. Ridha Mahjoub,,${ }^{c}$ Hande Yaman $^{b}$ \\ ${ }^{\mathbf{a}}$ HEC Montréal and CIRRELT, Montréal, Quebec H3T 2A7 Canada; ${ }^{\mathbf{b}}$ Department of Industrial Engineering, Bilkent University, Bilkent, \\ 06800 Ankara, Turkey; ${ }^{c}$ Université Paris Dauphine, PSL Research University, CNRS [7243], LAMSADE, 75016 Paris, Île-de-France, France \\ Contact: okan.arslan@hec.ca, (iD https://orcid.org/0000-0002-7862-3449 (OA); karasan@bilkent.edu.tr (OEK); \\ ridha.mahjoub@lamsade.dauphine.fr (ARM); hyaman@bilkent.edu.tr, (iD https://orcid.org/0000-0002-3392-1127 (HY)
}

Received: June 20, 2017

Revised: May 3, 2018

Accepted: August 9, 2018

Published Online in Articles in Advance: June 13, 2019

https://doi.org/10.1287/trsc.2018.0869

Copyright: @ 2019 INFORMS

\begin{abstract}
Because of the limited range of alternative fuel vehicles (AFVs) and the sparsity of the available alternative refueling stations (AFSs), AFV drivers cooperatively deviate from their paths to refuel. This deviation is bounded by the drivers' tolerance. Taking this behavior into account, the refueling station location problem with routing (RSLP-R) is defined as maximizing the AFV flow that can be accommodated in a road network by locating a given number of AFSs while respecting the range limitation of the vehicles and the deviation tolerance of the drivers. In this study, we develop a natural model for the RSLP-R based on the notion of length-bounded cuts, analyze the polyhedral properties of this model, and develop a branch-and-cut algorithm as an exact solution approach. Extensive computational experiments show that the algorithm significantly improves the solution times with respect to previously developed exact solution methods and extends the size of the instances solved to optimality. Using our methodology, we investigate the tradeoffs between covered vehicle flow and deviation tolerance of the drivers and present insights on deviation characteristics of drivers in a case study in California.

Funding: This work was partially supported by the Scientific and Technological Research Council of Turkey (TUBITAK) [Grant 214M211]. A. R. Mahjoub's research was partially supported by TUBITAK [Programme 2221].

Supplemental Material: The online appendix is available at https://doi.org/10.1287/trsc.2018.0869.
\end{abstract}

Keywords: alternative fuel vehicles $\cdot$ refueling station $•$ location $\cdot$ routing $\cdot$ branch-and-cut $•$ length-bounded cut

\section{Introduction}

The purpose of this paper is to develop a branch-andcut $(B \& C)$ algorithm for the refueling station location problem with routing (RSLP-R). In a transportation network with alternative fuel vehicles (AFVs) traveling between their origin-destination (OD) pairs, the RSLP$\mathrm{R}$ is defined as locating a given number of alternative fuel stations (AFSs) at the nodes of the network such that the total vehicle flow traveling without running out of fuel on paths whose lengths are bounded by the tolerance of the drivers is maximized. This problem is closely related with the efforts to curtail the negative impacts of fossil fuels on the environment. Dependence on fossil fuels contributes significantly to many of the environmental problems we face today, such as air pollution, globally increasing temperatures, and climate change. Because of their limited supply levels, reserves are destined to deplete. Historically, the transportation sector has been one of the major consumers of fossil fuels. However, there has been a recent surge for alternative forms of fuels to be used in transportation. These include hydrogen, biodiesel, electricity, ethanol, compressed natural gas, and liquefied natural gas (Energy Information Administration 2017a). In 2016, in the United States, 24.79 million passenger cars and light trucks using alternative energy sources were in use. This number accounts to $10.25 \%$ of the total number of the same vehicle types in the country, and it is projected to surpass $20 \%$ by 2030 (Energy Information Administration 2017b). The AFV usage in Europe is also following a similar trend. The Renewable Energy Directive (European Union 2009) set a 10\% target for use of energy from renewable sources in transportation by 2020, a considerable increase from the $4.7 \%$ target of 2013 (European Commission 2013).

Because of the rather limited range of AFVs, availability of refueling stations in intercity transportation is a serious barrier to the proliferation of these vehicles. In this regard, location of AFSs has been the topic of several recent articles. There have been two main streams of research, a set-covering location perspective (Wang and Lin 2009, 2013; Wang and Wang 2010) and a maximum covering location perspective. The latter attracted more attention because covering all the demand requires location of numerous facilities, which seems implausible in the initial setup period of the AFS infrastructure. To this end, Kuby and Lim (2005) presented the flow refueling location problem, which is defined 
as maximizing the AFV flow that can be accommodated in a road network by locating $p$ number of AFSs while respecting the range limitation of the vehicles. The demand is defined as the AFVs flowing on fixed paths between their OD pairs. "Flow refueling location problem" and "refueling station location problem" are used interchangeably in the literature to refer to the same problem (MirHassani and Ebrazi 2013, Y1ldiz et al. 2016). In this paper, we prefer to use the latter. Kuby and Lim (2005) proposed a maximal covering location model (MCLM; Church and ReVelle 1974), in which the coverage of an AFV flow requires location of possibly multiple facilities on its path. The formulation requires a priori generation of all node combinations that enable complete traversal of a path, which is computationally costly. For this reason, heuristic algorithms have been proposed (Lim and Kuby 2010).

Since then, considerable effort has been spent for improving the solution times and the size of the solvable RSLP instances. Two different exact solution approaches stand out in the literature. The first one, presented by MirHassani and Ebrazi (2013), builds on the idea that an AFV travels on the shortest path between two consecutive refueling stops. Given a fixed path, not necessarily the shortest, the authors first build a graph in which any path with a refueling station at all of its intermediate nodes corresponds to a trip in the original path that can be traveled without running out of fuel. Because of this key observation, no preprocessing is necessary to generate node combinations as in Kuby and Lim (2005), and the solution process is significantly accelerated. In the following section, we further elaborate on this transformation that has also been used by Chen et al. (2010) in the context of the regenerator placement problem. The second work, by Capar et al. (2013), refines the modeling logic using the fact that, to cover a path, every arc on this path needs to be traversed using one of the open stations. This new model is similar to the MCLM but does not require explicit generation of feasible node combinations to cover a path. Therefore, both the preprocessing and the solution times improve extensively. Further improvements on the solution times and the size of the solvable instances are obtained by applying Benders decomposition on this model (Arslan and Karaşan 2016).

In this study, we focus our attention on the RSLP-R introduced by Kim and Kuby (2012). In this version of the problem, the path between an OD pair is not fixed and an AFV flow is considered as refueled if there exists a feasible path whose length is within a certain bound. This bound is the tolerance of the driver to deviate from the shortest path. Kim and Kuby (2012) present a mixed integer programming formulation, Kim and Kuby (2013) propose a heuristic approach, and Yildız et al. (2016) present a branch-and-price (B\&P) algorithm to solve this problem. The computational gains of the $B \& P$ framework with respect to the original formulation by Kim and Kuby (2012) turned out to be significant.

Another closely related problem is the regenerator placement problem (Yetginer and Karasan 2003, Chen et al. 2010) defined in the context of telecommunications to locate the regenerators that extend the optical reach. From the functional perspective of extending the reach, the AFSs are similar to the regenerators. One major difference is related to the way demand is modeled. The drivers in our application have a distance tolerance on the deviation from the shortest path, which is not present in signal routing. This constitutes an additional challenge to our design problem. Computational findings in the regenerator placement problem show that $\mathrm{B} \& \mathrm{C}$ approaches outperform other exact solution methods, especially in large-scale instances (Rahman et al. 2015, Y1ldız and Karaşan 2015, Li and Aneja 2017). We also observe similar results.

In this paper, we propose a natural formulation for the RSLP-R and provide a polyhedral study of the convex hull of integer feasible solutions. We then devise a $B \& C$ algorithm as an exact solution technique to solve the problem. The constraints of our formulation, which are exponential in number, are added using a cuttingplane framework. The separation problem boils down to finding a length-bounded node cut in a transformed network. For separating integer solutions, we provide a polynomial time-exact separation algorithm to generate cuts. For fractional solutions, we provide a formulation to generate violated cuts. We also make use of a heuristic algorithm to separate fractional solutions. With our approach, we can solve real-world problem instances, which could only be solved previously by a B\&P algorithm in a three-hour time frame, within two minutes. We also further extend the size of the instances that can be solved to optimality. Our approach also addresses multiple vehicle types and possible nonsimple path occurrences in the routes. Using our methodology, we investigate the tradeoffs between covered vehicle flow and deviation tolerance of the drivers and present insights on deviation characteristics of drivers in a case study in California.

In the following, we introduce this new formulation, investigate its polyhedral properties, and present our $\mathrm{B} \& \mathrm{C}$ algorithm along with the results of our computational experiments.

\section{Definitions, Formulations, and Polyhedral Analysis}

Consider a road network represented by a weighted directed graph $G=(N, A)$ with node set $N=\{1, \ldots, n\}$ and arc set $A$. Let $\delta_{i j}$ be the shortest path distance in $G$ from node $i$ to node $j$. Suppose there are AFV drivers willing to travel between OD pairs in G. An AFV demand $q$ is defined as a five-tuple $\left\langle o_{q}, d_{q}, f_{q}, r_{q}, \lambda_{q}\right\rangle$, where 
$o_{q}$ and $d_{q}$ are the origin and destination nodes, respectively; $f_{q}$ is the flow volume; $r_{q}$ is the range of the vehicle; and $\lambda_{q}$ is the total distance that drivers can tolerate. With this definition, we can incorporate the vehicle flows between the same OD pair with different deviation tolerances. The set of demands is denoted by $Q$. Similar to previous studies, an AFV is assumed to depart from its origin with a half-full tank and is required to arrive at its destination at least with a half-full tank unless these nodes have AFSs. If there exists a station at the origin, then the AFV departs from the origin with a full tank. If the destination has a station, then the AFV can arrive at this node with an empty tank. The logic behind such an assumption is related to round trips between OD pairs; please refer to Kuby and Lim (2005) and MirHassani and Ebrazi (2013) for further details.

Definition 1. The RSLP-R is defined as finding a subset of $N$ with cardinality at most $p$ to locate the refueling stations such that the total amount of refueled vehicle flow respecting range and tolerance limitations is maximized.

A node is called a dominated node for a demand $q$ if it does not appear on any path from $o_{q}$ to $d_{q}$ of length at most $\lambda_{q}$. Note that we can identify whether a node $i$ is dominated by checking the shortest path distance between $o_{q}$ and $d_{q}$ using node $i$. Having $\delta_{o_{q} i}+\delta_{i d_{q}}>\lambda_{q}$ implies that node $i$ is a dominated node for demand $q$. Next, we adapt the network transformation of MirHassani and Ebrazi (2013) to the case in which OD paths are not fixed. For each $q \in Q$, consider graph $G_{q}=\left(N_{q}, A_{q}\right)$ with node set $N_{q}=\left\{s_{q}, t_{q}\right\} \cup\left\{i \in N: \delta_{o_{q} i}+\right.$ $\left.\delta_{i d_{q}} \leq \lambda_{q}\right\}$, where $s_{q}$ and $t_{q}$ are two new dummy nodes, and arc set $A_{q}=A_{q}^{1} \cup A_{q}^{2} \cup A_{q}^{3}$, where

$$
\begin{aligned}
& A_{q}^{1}=\left\{\left(s_{q}, j\right): \delta_{o_{q} j} \leq r_{q} / 2, j \in N_{q} \backslash\left\{s_{q}, t_{q}\right\}\right\}, \\
& A_{q}^{2}=\left\{\left(i, t_{q}\right): \delta_{i d_{q}} \leq r_{q} / 2, i \in N_{q} \backslash\left\{s_{q}, t_{q}\right\}\right\}, \\
& A_{q}^{3}=\left\{(i, j): \delta_{i j} \leq r_{q}, i, j \in N_{q} \backslash\left\{s_{q}, t_{q}\right\}, i \neq j\right\} .
\end{aligned}
$$

$\operatorname{Arc}\left(s_{q}, j\right) \in A_{q}^{1}$ has length $\delta_{o_{q}}$, arc $\left(i, t_{q}\right) \in A_{q}^{2}$ has length $\delta_{i d_{q}}$, and $\operatorname{arc}(i, j) \in A_{q}^{3}$ has length $\delta_{i j}$. Each arc in the transformed graph corresponds to traveling on the shortest path in the underlying road network between the tail and the head nodes of the arc without refueling. The dummy nodes $s_{q}$ and $t_{q}$ are added to model the refueling logic, and they represent departing from the origin with a half-full tank and arriving at the destination with at least a half-full tank. Therefore, the arcs emanating from $s_{q}$ (i.e., arcs in set $A_{q}^{1}$ ) and the arcs entering to $t_{q}$ (i.e., arcs in set $A_{q}^{3}$ ) have length at most $r_{q} / 2$. Refueling at the origin node is represented by arc $\left(s_{q}, o_{q}\right)$. Traversing this arc is identified with the AFV departing from node $o_{q}$ with a full tank. The same logic also applies to the destination node. All nodes except the first and the last nodes on a given path are referred
Figure 1. Length-Bounded Cut Example

(a)

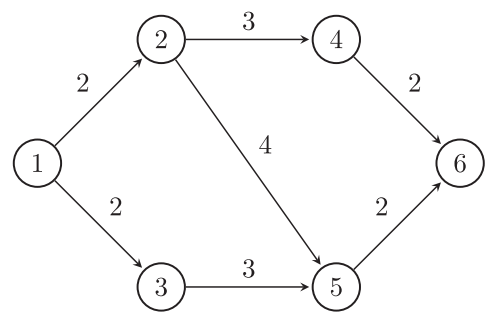

Graph representation of the road network

(b)

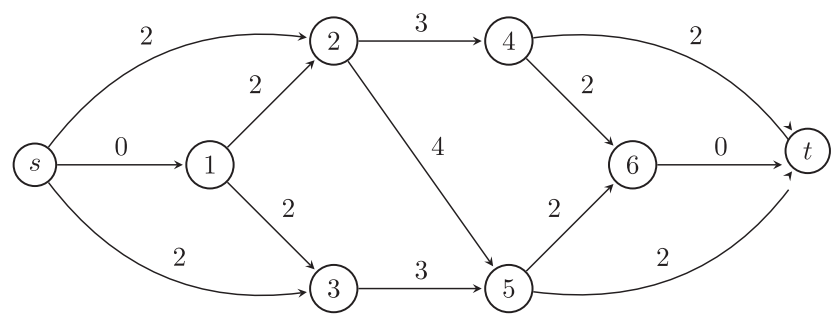

Transformed graph

to as the path's internal nodes. By assumption, an AFV departs from $s_{q}$ with a half-full tank, and no station is required to traverse an arc emanating from this node. By construction, all other arcs in the transformed graph can be traversed if an AFS is located at the tail node of the arc. Therefore, to satisfy a given demand $q$, there should exist a path in $G_{q}$ of distance at most $\lambda_{q}$ from $s_{q}$ to $t_{q}$ with a refueling station at each of its internal nodes. We refer to such a path as feasible.

For a demand $q \in Q$, let $\mathscr{P}_{q}$ be the set of all directed paths in $G_{q}$ from $s_{q}$ to $t_{q}$ with lengths at most $\lambda_{q}$. A subset of nodes $S \subseteq N_{q} \backslash\left\{s_{q}, t_{q}\right\}$ is called a q-node-cut for path set $\mathscr{P}_{q}$ if $S$ intersects each path in $\mathscr{P}_{q}$ at a node different from $s_{q}$ and $t_{q}$; in other words, removing $S$ from the set of nodes disconnects $s_{q}$ and $t_{q}$. A $q$-nodecut $S$ is called minimal for $\mathscr{P}_{q}$ if no proper subset of $S$ is a $q$-node-cut for $\mathscr{P}_{q}$. Let $\Gamma_{q}$ represent the set of all $q$-node-cuts for $\mathscr{P}_{q}$.

Figure 1, (a) and (b), displays a graph representation of an example road network and its transformed graph, respectively. Consider a demand $q$ with $o_{q}=1, d_{q}=6$, $r_{q}=4$, and $\lambda_{q}=7$. The cuts $\{2,3\},\{2,5\},\{3,4\}$, and $\{4,5\}$ in the transformed graph are all minimal $q$-nodecuts for $\mathscr{P}$. Note that although cuts $\{2,3\},\{2,5\}$, and $\{4,5\}$ disconnect all paths from $s_{q}$ to $t_{q}$, cut $\{3,4\}$ disconnects only the length-bounded paths, and the $s_{q}-(1)$ $2-5-(6)-t_{q}$ path remains connected.

\subsection{Natural Formulation}

In this section, we propose a formulation based on the notion of $q$-node-cuts. The model has the following variables: 
$x_{i}= \begin{cases}1, & \text { if there is arefueling station located at node } \\ & i \in N \\ 0, & \text { otherwise; }\end{cases}$ $y_{q}= \begin{cases}1, & \text { if afeasible path is constructed for demand } \\ & q \in Q \\ 0, & \text { otherwise. }\end{cases}$

We refer to $x$ as location variables and $y$ as cover variables. For convenience, we use $x(S)=\sum_{i \in S} x_{i}$ for set $S \subseteq N$. We formulate the RSLP-R as follows:

$$
\begin{aligned}
& \max \sum_{q \in Q} f_{q} y_{q} \\
& \text { s.t. } x(N) \leq p \text {, } \\
& y_{q} \leq x(S) \quad \forall q \in Q, S \in \Gamma_{q}, \\
& x_{i} \in\{0,1\} \quad \forall i \in N \text {, } \\
& y_{q} \in\{0,1\} \quad \forall q \in Q .
\end{aligned}
$$

The objective function maximizes the total vehicle flow refueled. Constraint (2) ensures that at most $p$ stations are located. Constraints (3) express the fact that for having a feasible trip for demand $q$, the corresponding transformed graph needs to be connected from $s_{q}$ to $t_{q}$ by at least one path of length at most $\lambda_{q}$ on which every internal node has a refueling station. Suppose that, for demand $q$, there exists no such path. Then there exists a subset $S$ of $N_{q} \backslash\left\{s_{q}, t_{q}\right\}$ such that $S$ contains at least one node from each path in $\mathscr{P}_{q}$. If none of the nodes in $S$ has a station, constraints (3) for this choice of $S$ force $y_{q}$ to zero because $q$ cannot be refueled. Constraints (4) and (5) are integrality constraints. Note that one can relax integrality of the cover variables without changing the optimal value. One of the main advantages of this model is that we have natural variables associated with only the nodes and OD pairs.

The idea of $q$-node-cuts can easily be extended to the set-covering version of the problem, in which one seeks to find the minimum number of AFSs to satisfy all the demand.

$$
\begin{aligned}
& \min \sum_{i \in N} x_{i} \\
& \text { s.t. } x(S) \geq 1 \quad \forall q \in Q, S \in \Gamma_{q} \text {, } \\
& x_{i} \in\{0,1\} \quad \forall i \in N \text {. }
\end{aligned}
$$

The objective function minimizes the number of selected refueling stations. Constraints (7) ensure that, for every demand, all $q$-node-cuts have at least one selected refueling station, which implies that there exists a length-bounded path between every OD pair. Constraints (8) are variable restrictions.

The budget for AFSs is usually limited. For this reason, we consider the maximum-covering version of the problem in this paper.

\subsection{Polyhedral Analysis}

Let $\mathscr{X}$ be the feasible set of the natural formulation given by (2)-(5). In the following, we assume that $|N| \geq 2$. For a path $\pi \in \mathscr{P}_{q}$, let $N(\pi)$ be the set of its internal nodes. We define, for each $q \in Q$, set $\mathscr{P}_{q}^{\prime}$ to be the set of paths in $\mathscr{P}_{q}$ with at most $p$ internal nodes. We assume, without loss of generality, that $\mathscr{P}_{q}^{\prime}$ contains at least one path for all $q \in Q$ (otherwise, $y_{q}=0$ in all feasible solutions, and demand $q$ can be removed from set $Q$ ).

Let $\operatorname{conv}(\mathscr{X})$ be the convex hull of all the solutions in $\mathscr{X}$. In this section, we study the polyhedral properties of $\operatorname{conv}(\mathscr{X})$ and prove that most of the constraints of the natural formulation are facet-defining inequalities under some conditions. The proofs of Propositions 1 through 6 are presented in the online appendix.

Proposition 1. The convex hull of $\mathscr{X}$ is full dimensional.

Next, we give necessary and sufficient conditions for the trivial inequalities to be facet defining.

Proposition 2. For $q \in Q$, the inequality $y_{q} \geq 0$ is facet defining for conv( $\mathscr{X})$.

Proposition 3. For $i \in N$, the inequality $x_{i} \geq 0$ is facet defining for conv(X) if and only if $\{i\}$ is not a q-node-cut for $\mathscr{P}_{q}^{\prime}$ for all $q \in Q$.

Proposition 4. For $i \in N$, the inequality $x_{i} \leq 1$ is facet defining for conv( $\mathscr{X})$ if and only if $p \geq 2$ and there exists a path $\pi^{q} \in \mathscr{P}_{q}^{\prime}$ such that $\left|N\left(\pi^{q}\right) \cup\{i\}\right| \leq p$ for all $q \in Q$.

In the next two propositions, we put forward the conditions under which the other constraints of the model are facet defining.

Proposition 5. The inequality $x(N) \leq p$ is facet defining for conv(囚) if and only if $p<|N|$.

Proposition 6. For $q \in Q$ and a q-node-cut $S$ that is minimal for path set $\mathscr{P}_{q}^{\prime}$, the inequality $y_{q} \leq x(S)$ is facet defining for conv( $\mathscr{X})$ if and only if, for every $\hat{q} \in Q \backslash\{q\}$, either there exists a path $\pi^{\hat{q}} \in \mathscr{P}_{\hat{q}}^{\prime}$ with $N\left(\pi^{\hat{q}}\right) \cap S=\emptyset$ or there exist a node $i \in S$, a path $\pi^{q} \in \mathscr{P}_{q^{\prime}}^{\prime}$, and a path $\pi^{\hat{q}} \in \mathscr{P}_{\hat{q}}^{\prime}$ such that $N\left(\pi^{q}\right) \cap$ $S=N\left(\pi^{\hat{q}}\right) \cap S=\{i\}$ and $\left|N\left(\pi^{q}\right) \cup N\left(\pi^{\hat{q}}\right)\right| \leq p$.

\section{Separation Problem}

In our formulation, constraints (3) are exponential in number, and we need a cutting plane algorithm to generate them. For a given solution $\left(x^{*}, y^{*}\right)$ and a demand $q \in Q$ with $y_{q}^{*}>0$, the separation problem is to identify a $q$-node-cut $S \subseteq N_{q} \backslash\left\{s_{q}, t_{q}\right\}$ for path set $\mathscr{P}_{q}$ with $x^{*}(S)<y_{q}^{*}$ or to conclude that none exists.

\subsection{Separating Integer Solutions}

Consider an integer solution $\left(x^{*}, y^{*}\right)$. For a subset of nodes $N^{\prime} \subseteq N$, we define $G_{q}\left(N^{\prime}\right)$ to be the subgraph of $G_{q}$ induced by nodes in $N^{\prime}$. For each $q \in Q$ with $y_{q}^{*}=1$, we compute the length of the shortest path from $s_{q}$ to $t_{q}$ 
in $G_{q}\left(N_{q}^{*}\right)$, where $N_{q}^{*}=\left\{s_{q}, t_{q}\right\} \cup\left\{i \in N_{q}: x_{i}^{*}=1\right\}$. If the shortest path length is less than or equal to $\lambda_{q}$, then the solution contains a feasible path for demand $q$. If not, then at least one station needs to be located at those nodes with $x_{i}^{*}=0$ to refuel demand $q$. In other words, inequality (3) for the $q$-node-cut $N_{q} \backslash N_{q}^{*}$ is violated. We refer to the process of identifying such a $q$-node-cut as IntSep.

Clearly, $N_{q} \backslash N_{q}^{*}$ may not be a minimal $q$-node-cut for path set $\mathscr{P}_{q}$. For a node $i \in N_{q} \backslash N_{q}^{*}$, if the shortest path from $s_{q}$ to $t_{q}$ in $G_{q}\left(N_{q}^{*} \cup\{i\}\right)$ is greater than $\lambda_{q}$, then locating a station at node $i$ cannot render the demand feasible, and $N_{q} \backslash\left(N_{q}^{*} \cup\{i\}\right)$ is also a $q$-node-cut for $\mathscr{P}_{q}$. The associated inequality (3) for this new $q$-node-cut dominates the former one. We can repeat this operation until a minimal $q$-node-cut is attained. When the cuts generated by IntSep are also minimalized, then the process is referred to as IntSep-M. In the following section, we show that strengthening the generated cuts in this fashion proved to be highly effective in reducing the computational times.

\subsection{Separating Fractional Solutions}

Now suppose that $\left(x^{*}, y^{*}\right)$ is fractional. Consider a demand $q \in Q$ with $y_{q}^{*}>0$. We define the minimum weight $q$-node-cut problem $(\mathrm{MqCP})$ as follows: given graph $G_{q}$ with node weight $x_{i}^{*}$ for node $i \in N_{q} \backslash\left\{s_{q}, t_{q}\right\}$, find a minimum weight subset $S^{*}$ of $N_{q} \backslash\left\{s_{q}, t_{q}\right\}$ such that deleting the nodes in $S^{*}$ disrupts all directed paths from node $s_{q}$ to node $t_{q}$ with lengths of at most $\lambda_{q}$. There exists an inequality (3) for demand $q$ that is violated by $\left(x^{*}, y^{*}\right)$ if and only if $x^{*}\left(S^{*}\right)<y_{q}^{*}$.

The special case of MqCP in which all arcs have unit lengths is called the length-bounded minimum node-cut problem (Lovász et al. 1978), which is known to be NPhard for lengths greater than four units (Baier et al. 2006, Mahjoub and McCormick 2010).

Consider a variable $u_{i}$, which equals one if node $i \in$ $N_{q} \backslash\left\{s_{q}, t_{q}\right\}$ is in the $q$-node-cut and zero otherwise. We also define $\pi_{i}$ to be the length of a shortest path from node $i \in N_{q}$ to node $t_{q}$ in graph $G_{q}\left(N_{q}^{*}\right)$, where $N_{q}^{*}=$ $\left\{s_{q}, t_{q}\right\} \cup\left\{i \in N_{q} \backslash\left\{s_{q}, t_{q}\right\}: u_{i}=0\right\}$. We let $M$ be a very large number and $\varepsilon$ a very small positive number. We refer to the following model as the minimum weight q-node-cut model (MqCM):

$$
\begin{array}{cc}
(\mathrm{MqCM}) \min & \sum_{i \in N_{q} \backslash\left\{s_{q}, t_{q}\right\}} x_{i}^{*} u_{i} \\
\text { s.t. } \pi_{t_{q}}=0, u_{t_{q}}=0, &
\end{array}
$$

Table 1. B\&C Algorithm Implementations

\begin{tabular}{lcc}
\hline & \multicolumn{2}{c}{ Separation algorithm } \\
\cline { 2 - 3 } Implementation & Integer solutions & Fractional solutions \\
\hline B\&C-1 & IntSep-M & \\
B\&C-2 & IntSep-M & MqCM \\
B\&C-3 & IntSep-M & MinCut \\
B\&C-4 & IntSep & \\
B\&C-5 & IntSep-M & MinCut-M \\
\hline
\end{tabular}

The objective function minimizes the total weight of nodes in the node cut. Constraint (10) sets the shortest path length from $t_{q}$ to itself to zero, and it forbids this node to be in the node cut. Constraints (11) ensure that $\pi_{i}$ is not more than the length of a shortest path from node $i$ to node $t_{q}$ in the graph obtained by removing the nodes with $u_{j}=1$. The shortest path length from $s_{q}$ to $t_{q}$ after these nodes are removed is forced to be greater than $\lambda_{q}$ by constraint (12). Constraints (13) and (14) are the nonnegativity and integrality constraints.

In the next section, we present computational results in which we use this model for separation. However, this turns out to be computationally costly in most cases. For this reason, we also propose a simple and efficient heuristic approach. Observe that any node cut that disconnects $s_{q}$ and $t_{q}$ is also a $q$-node-cut for set $\mathscr{P}_{q}$. For all $q \in Q$, we search for the minimum weight node cut in graph $G_{q}$ and add the corresponding cut if a violation is identified. To find a node cut $S$, we split every node $i \in N_{q} \backslash\left\{s_{q}, t_{q}\right\}$ into two nodes $i^{\prime}$ and $i^{\prime \prime}$ and add arc $\left(i^{\prime}, i^{\prime \prime}\right)$ for every such $i$. Those arcs entering into node $i$ will be entering into node $i^{\prime}$, and those arcs leaving node $i$ will be leaving node $i^{\prime \prime}$. All the arcs have infinite capacity except for those that represent nodes (i.e., arcs of the form $\left.\left(i^{\prime}, i^{\prime \prime}\right)\right)$. Arc $\left(i^{\prime}, i^{\prime \prime}\right)$ has capacity equal to $x_{i}^{*}$. Solving a minimum cut problem on this transformed network gives a cut with the minimum $x^{*}(S)$ value. If $x^{*}(S)<y_{q}^{*}$, then a violated cut is identified that separates a fractional or integer infeasible solution at hand. We refer to this process as the mincut heuristic. Note that the minimum node cuts we obtain by the heuristic are not necessarily minimal $q$-node-cuts because they disconnect all paths regardless of their lengths. Therefore, we can strengthen the generated cuts by the cut minimalization process, similar to the logic in integer separation. We refer to the heuristic as mincut-M if the cuts generated by the mincut are minimalized.

\section{Computational Study}

We propose a B\&C algorithm to solve the RSLP-R because constraints (3) are exponential in number. We tested five different implementations (Table 1). The first one, which we refer to as $B \mathcal{E} C$ - 1 , uses separation only at integer solutions. In the second and third implementations, we separate both integer and fractional solutions. We 
Table 2. Characteristics of Instances

\begin{tabular}{|c|c|c|c|c|c|c|c|c|}
\hline \multirow[b]{2}{*}{ Network } & \multirow[b]{2}{*}{ Number of nodes } & \multirow[b]{2}{*}{ Number of arcs } & \multicolumn{3}{|c|}{ Node degree } & \multicolumn{3}{|c|}{ OD pairs } \\
\hline & & & Minimum & Mean & Maximum & Minimum distance & Mean distance & Maximum distance \\
\hline CA & 339 & 1,234 & 2 & 3.64 & 14 & 30.06 & 153.37 & 463.50 \\
\hline G-250 & 250 & 636 & 2 & 5.09 & 14 & 30.02 & 138.93 & 389.00 \\
\hline G-500 & 500 & 1,284 & 2 & 5.14 & 20 & 30.05 & 142.95 & 366.72 \\
\hline G-750 & 750 & 1,922 & 2 & 5.13 & 16 & 30.00 & 153.87 & 522.43 \\
\hline G-1000 & 1,000 & 2,580 & 2 & 5.16 & 22 & 30.00 & 160.98 & 458.86 \\
\hline
\end{tabular}

use the $\mathrm{MqCM}$ in $\mathrm{B} \& \mathrm{C}-2$ and the mincut heuristic in $B \& C-3$ to separate fractional solutions. B\&C-4 and B\&C-5 are designed to test the efficiency of the cut minimalization process. In $B \& C-4$, we only separate integer solutions, similar to $B \& C-1$, but without minimalizing the generated cuts. $B \& C-5$, by contrast, is designed to test whether the cut minimalization can also help in strengthening the cuts we obtain by the mincut heuristic. In B\&C-5, we separate both integer and fractional solutions, similar to $B \& C-3$, with the only difference that the cuts generated by the mincut heuristic are minimalized.

For branching, we use the default settings of CPLEX. We turn off the CPLEX cuts as our preliminary analysis showed that this gives shorter computation times.

Extensive computational experiments are carried out to test the efficiency of the proposed $B \& C$ algorithms. The experiments are executed using CPLEX 12.6.1 (IBM 2014), implemented in a Java programming environment under Linux using Concert Technology. The computer has an Intel Xeon E5-2630 v2 processor at $2.60 \mathrm{GHz}$ and $96 \mathrm{~GB}$ of RAM. The algorithms are implemented using callback classes. A time limit of one hour is set in all implementations.

Figure 2. (Color online) California Road Network

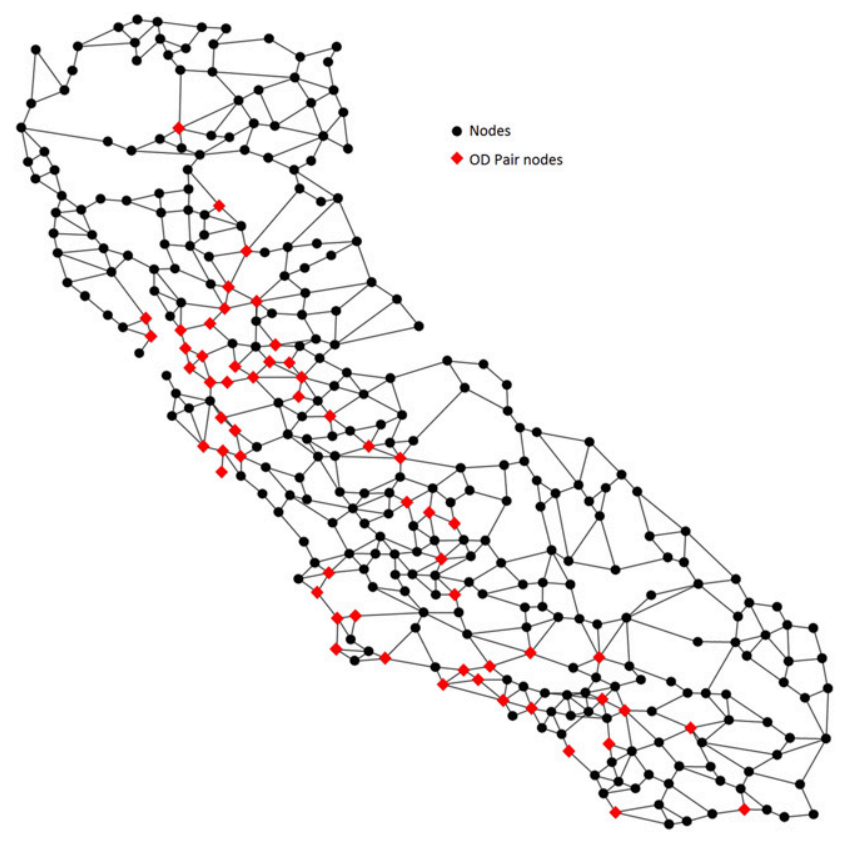

Characteristics of network topologies considered in this study are detailed in Table 2. CA is a real-world representation of the California road network with 339 nodes and 1,234 arcs, as shown in Figure 2 (Arslan et al. 2014). The nodes of the network represent road intersections or urban population centers. Similar to previous studies, all urban centers with a population of 50,000 or more are selected as origin or destination nodes, which are depicted in Figure 2 as OD pair nodes. There are 1,167 OD pairs, and they are 30 kilometers or more apart from each other. The vehicle flow volume between each OD pair is calculated according to the gravity model by Hodgson (1990). Networks G-250, G-500, G-750, and G-1000 are randomly generated networks with 250, 500, 750, and 1,000 nodes, respectively. To generate random graphs, we use JGraphT Java graph library (Naveh et al. 2008). Arc lengths are generated from a uniform distribution on the interval $(0,50)$ kilometers. Triangular inequality is not considered. For these graphs, we randomly select nodes with equal probabilities to represent origins or destinations. Similar to the CA network, we consider those OD pairs that are 30 kilometers or more apart. The number of OD pairs changes between experiments and varies between 1,000 and 4,000 .

We mainly compare our results with the results of the B\&P algorithm by Yildiz et al. (2016). Kim and Kuby (2013) present a heuristic algorithm based on network transformation; however, because the refueling station location problem is strategic in nature, we prefer to compare only with the exact solution methods in the literature. To this end, a computational comparison of the model by Kim and Kuby (2012) with a B\&P algorithm is previously presented by Yildiz et al. (2016), and it is shown that the former model cannot scale up to large networks because of enumeration requirements.

Let $\hat{\lambda}_{q}=100 \times \lambda_{q} / \delta_{o_{q}, d_{q}}$ for all $q \in Q$ be the deviation tolerance as a percentage of the shortest path length. Note that our model is capable of handling different driver tolerances for each OD pair. Furthermore, our demand definition allows us to model varying tolerances between the same OD pair. However, as in the previous studies, we assume equal deviation tolerance percentage for all demand, which we refer to as $\hat{\lambda}=\hat{\lambda}_{q}, q \in Q$. 


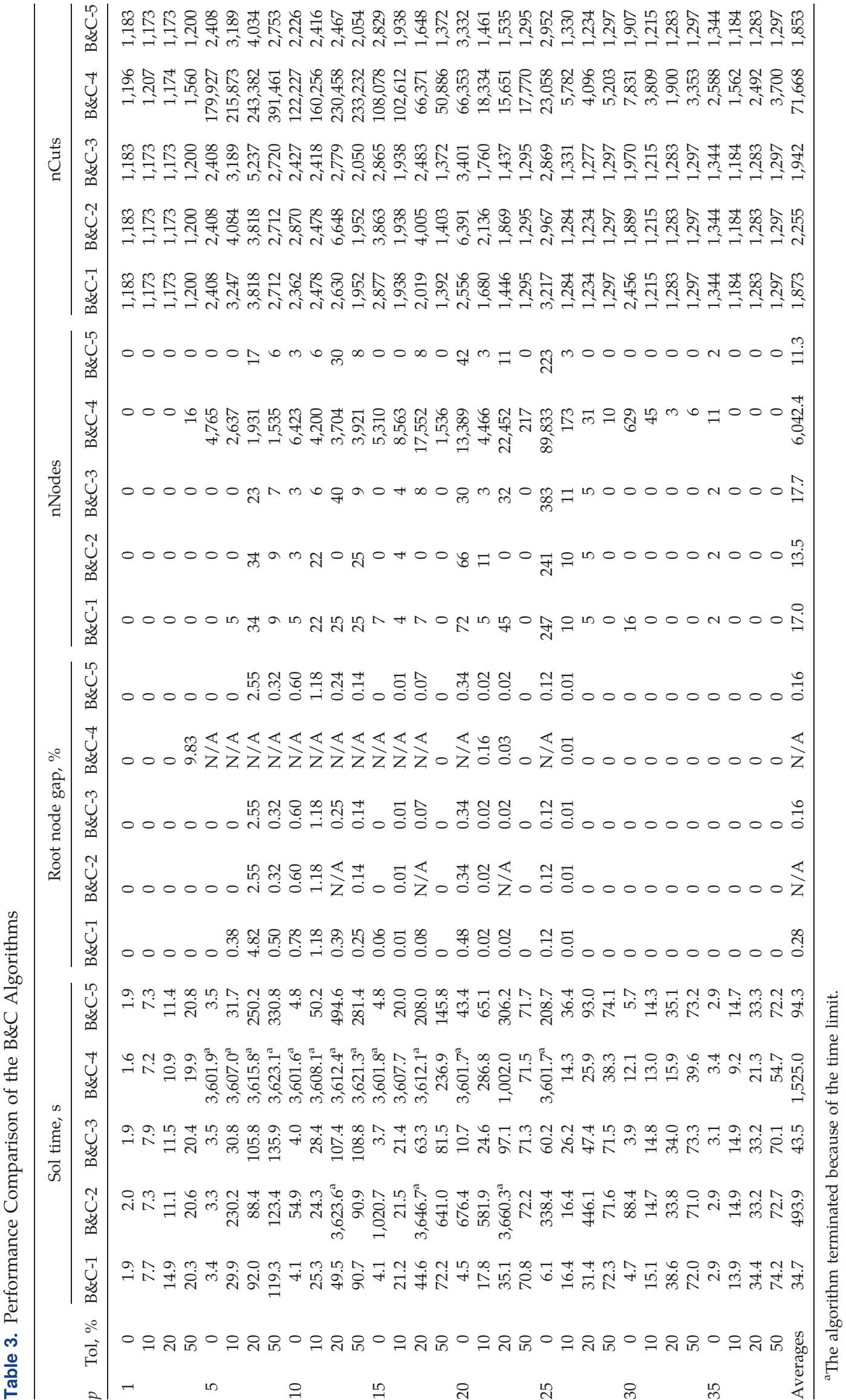


Table 4. Performance Summaries of B\&C Algorithms

\begin{tabular}{lrcccccc}
\hline & \multicolumn{3}{c}{ Average time, s } & & \multicolumn{2}{c}{ Nodes removed, \% } \\
\cline { 2 - 4 } Implementation & \multicolumn{1}{c}{ Total } & Integer & Fractional & AvgNCuts & & IntSep-M & MinCut-M \\
\hline B\&C-1 & 34.7 & 34.3 & 0 & 1,873 & 65.11 & - \\
B\&C-2 & 493.9 & 19.0 & 473.9 & 2,255 & 64.82 & - \\
B\&C-3 & 43.5 & 30.9 & 12.2 & & 1,942 & 64.71 & - \\
B\&C-4 & $1,525.0$ & 43.6 & 0 & & 71,668 & & - \\
B\&C-5 & 94.3 & 28.7 & 65.2 & & 1,853 & 64.75 & 17.66 \\
\hline
\end{tabular}

In the following, we first compare the five $\mathrm{B} \& \mathrm{C}$ algorithms on the CA network instances. This network is the largest-size network used in testing the B\&P algorithm in Yildiz et al. (2016). We then increase the number of demands and the size of the networks to investigate the limitations of our approach.

\subsection{California Network}

Our first objective is to compare five $B \& C$ implementations in terms of solution times using this large network. For this purpose, we consider a vehicle with a range of 100 kilometers. As in Yildiz et al. (2016), the number of stations considered are $1,5,10, \ldots, 35$, and the drivers are assumed to be $0 \%, 10 \%$, and $20 \%$ tolerant to deviating from their shortest paths. Different from the previous settings, we also consider deviations of 50\% in our experimental design. The results are presented in Table 3. The two leftmost columns are parameters of the experiment: $p$ is the number of stations to be located, and "Tolerance, $\%$ " is the drivers" deviation tolerance from the shortest path as a percentage. For instance, 10\% tolerance means that the drivers are willing to drive up to $10 \%$ more from their shortest paths. The following five columns in the table show the solution times in seconds. "Root node gap" is the percentage gap between the upper bound (UB) at the root node and the optimal value, calculated as $(\mathrm{UB}-\mathrm{OPT}) / \mathrm{OPT} \times 100 \%$. Note that for the B\&C-2 algorithm, the upper bound we obtain at the root node is equal to the optimal value of the linear programming (LP) relaxation of the model because we use exact algorithms for separation of both integer and fractional solutions and turn off the presolve and CPLEX cuts. "nNodes" is the number of nodes in the search tree. The rightmost five columns show the number of user cuts added by the algorithms.

The average solution times are 34.7, 493.9, 43.5, 1,525.0, and 94.3 seconds for the B\&C- 1 through B\&C-5 algorithms, respectively. In Table 4, the time for separating integer and fractional solutions are shown in columns (3) and (4), respectively. The average number of cuts added ( $A v g N C u t s)$ is reported in column (5). The average percentage of nodes removed from cuts by the minimalization algorithm for integer separation (IntSep-M) and the mincut heuristic (MinCut-M) are also reported in the table.
The B\&C-2 algorithm could not find the optimal solution of three instances (marked in Table 3) within the one-hour time limit; it terminated at the root node, and the maximum optimality gap was $3.1 \%$. Table 4 shows that the B\&C-2 algorithm spends more than $95 \%$ of the time for the fractional separation at the root node; however, no significant improvements can be achieved over the other algorithms in terms of root node gap. In other words, the time that the B\&C-2 algorithm spends to separate inequalities (3) exactly does not pay off. Furthermore, the unpredictable solution times of the $\mathrm{MqCM}$ model to separate fractional solutions in B\&C-2 cause extended solution times in several instances, and therefore, the solution times do not follow an obvious pattern in Table 3. Next, we compare the B\&C-1 and $\mathrm{B} \& \mathrm{C}-4$ algorithms and observe that the cut-minimalizing algorithm is highly effective in reducing the computation times. Without minimalization, the B\&C-4 algorithm spends less than $3 \%$ of the time for solving the separation problem, mainly because of weak cuts being added. Therefore, it fails to solve 13 of the 32 instances within the time limit (Table 3). In B\&C-1, by contrast, the minimalization process removes, on average, $65.11 \%$ of the nodes from the cuts generated by the IntSep-M algorithm (Table 4). Finally, we compare the B\&C-3 and B\&C-5 algorithms to see the effects of cut minimalization in the cuts generated by the mincut heuristic. In $B \& C-5$, on average, minimalization removes $17.66 \%$ of the nodes from the cuts generated by the heuristic; however, this came at a cost of multiplying the fractional separation times by more than five. The average time for fractional separation increased from 12.2 to 65.2 seconds. Even though the average number of cuts added is reduced from 1,942 to 1,853 , the average solution time increased from 43.5 seconds in B\&C-3 to 94.3 seconds in $B \& C-5$. The main reason for the cut minimalization to perform well in the IntSep algorithm but not in the mincut heuristic is that the cuts generated by the mincut heuristic are already small in size. However, the size of $q$-node-cut generated by the IntSep algorithm is large because all those nodes without a refueling station are in the cut. Thus, more nodes are removed from the cuts generated by the IntSep algorithm, and the time spent for cut minimalization pays off. According to the performance results, 


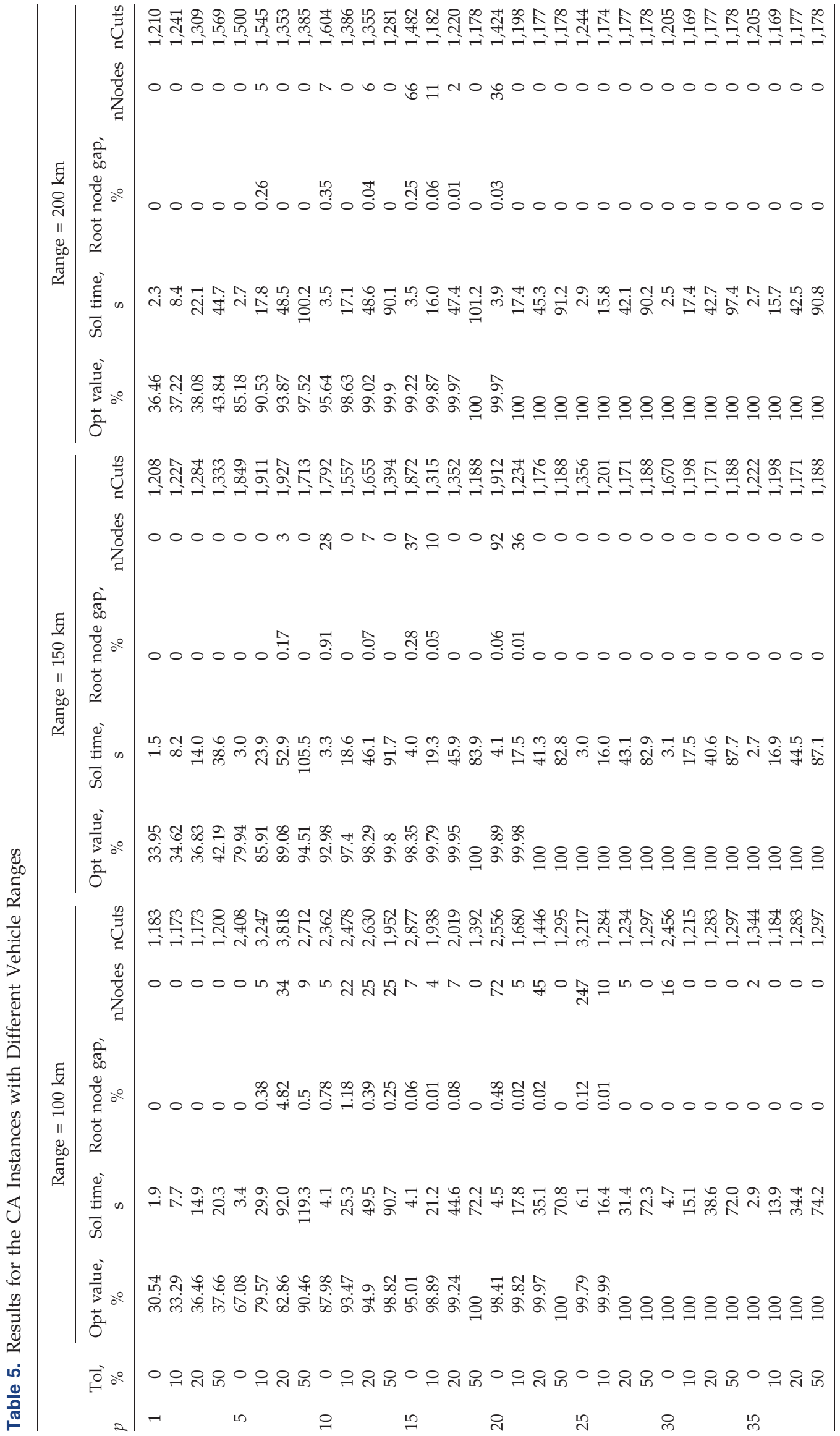


Figure 3. (Color online) Covered Flow as a Percentage of the Total Vehicle Flow for $p=1, \ldots, 35$ for (a) Range $=100 \mathrm{~km}$, (b) Range $=150 \mathrm{~km}$, and (c) Range $=200 \mathrm{~km}$ in the CA network

(a)

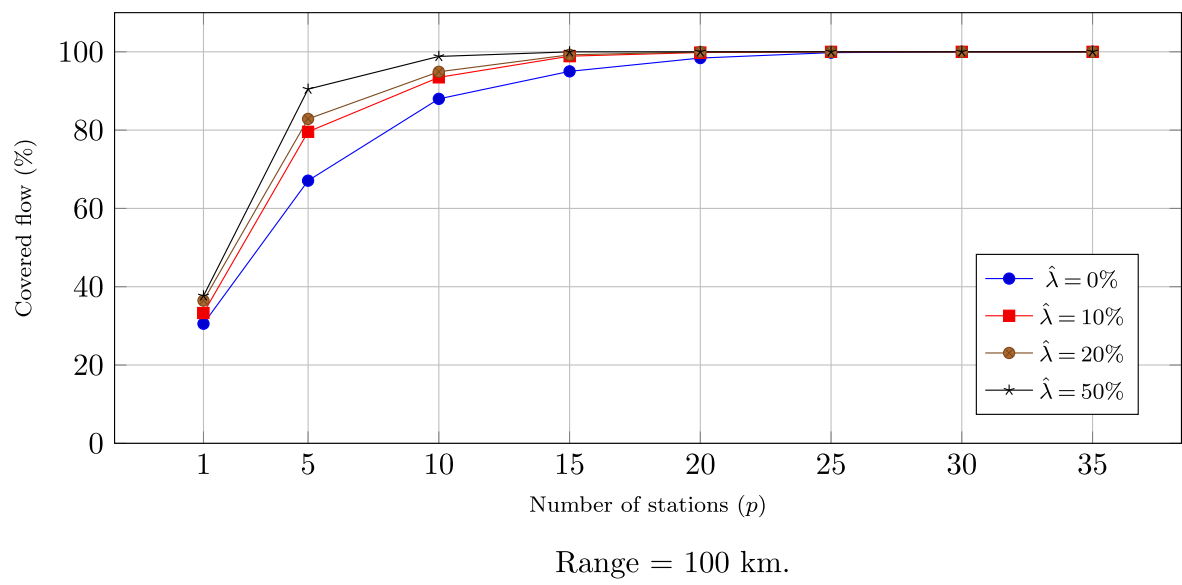

(b)

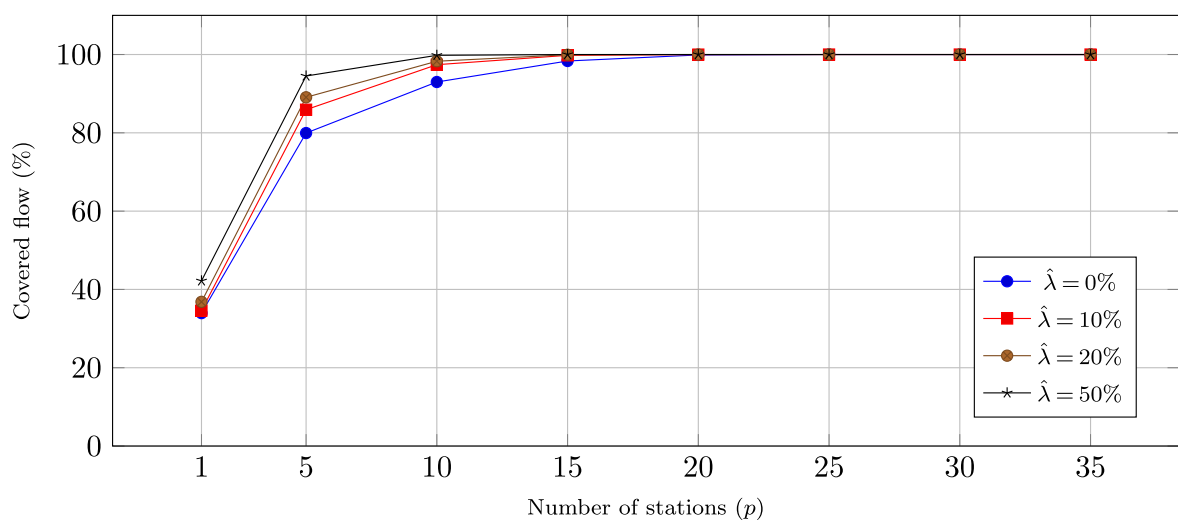

Range $=150 \mathrm{~km}$.

(c)

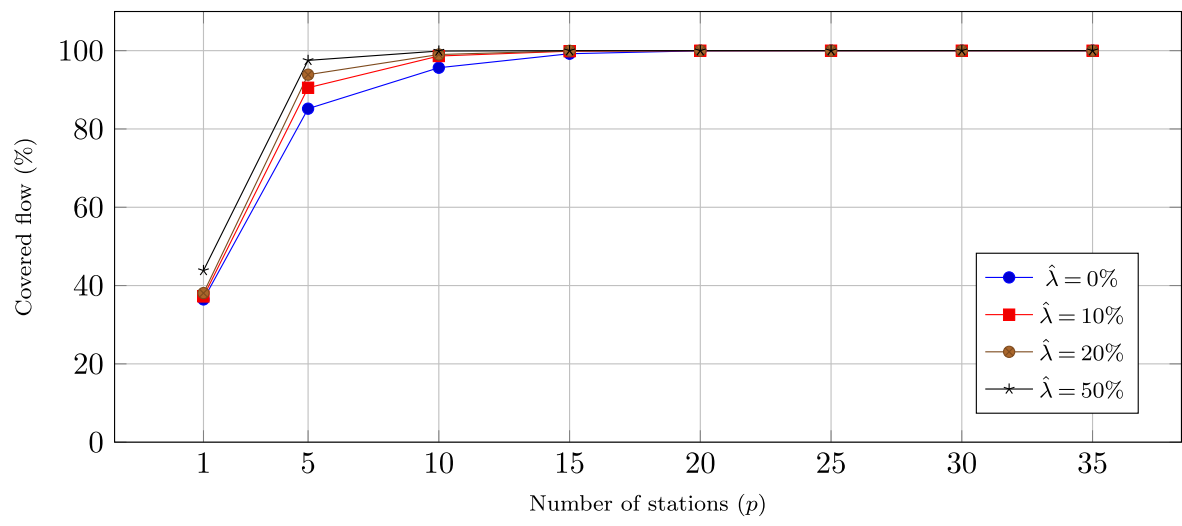

Range $=200 \mathrm{~km}$.

the $\mathrm{B} \& \mathrm{C}-1$ algorithm, implementing separation only at integer solutions and the cut minimalization algorithm, performs the best of the five versions. It is more than 14 times faster than the second implementation. The root node gaps of the second and third imple- mentations are the same for all the instances in which both could finish solving the LP relaxation within the time limit. With the best solution times and very small root node gaps, we perform our further analyses using the B\&C-1 algorithm. 
Figure 4. (Color online) (a) Percentage of Covered and Missed Vehicle Flows for Different Driver Tolerances in the California Network with Vehicles of 100-km Range and When $p=5$ Stations Are Optimally Located, (b) Percentage of Covered and Missed Vehicle Flows When $p=1, \ldots, 35$ Stations Are Optimally Located in the California Network with Vehicles of 100-km Range and for Deviation Tolerance $\hat{\lambda}=10 \%$

(a)

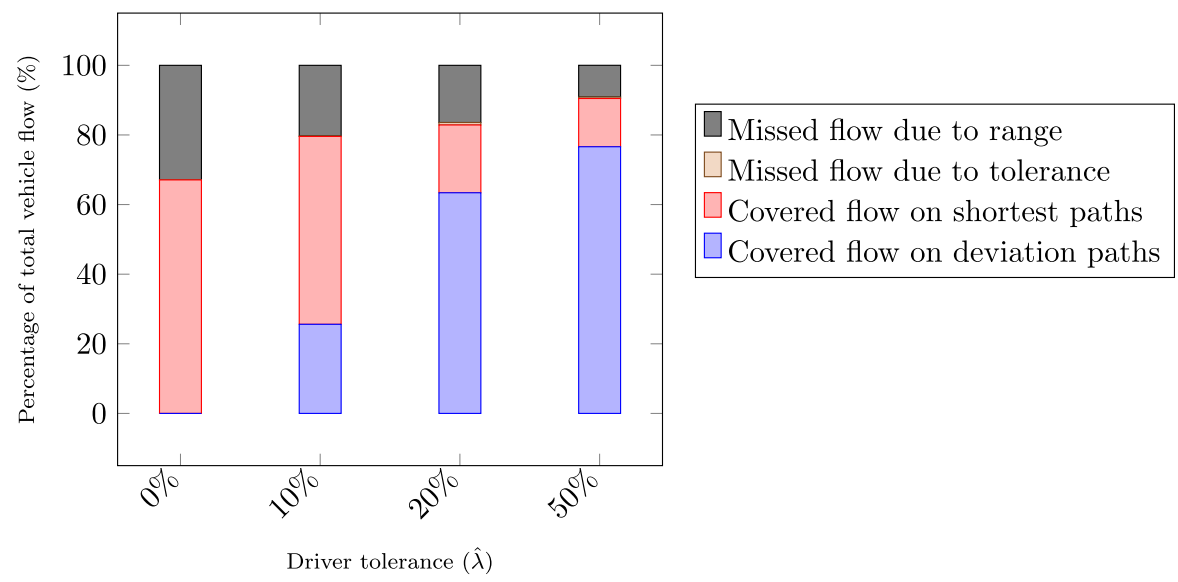

(b)

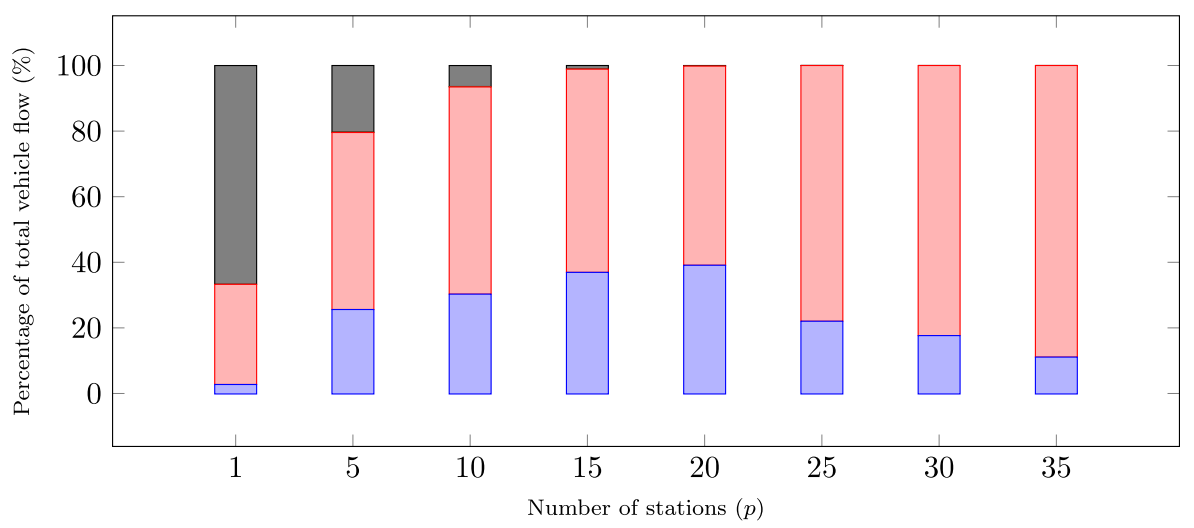

Detailed results with the $\mathrm{B} \& \mathrm{C}-1$ algorithm and the $\mathrm{CA}$ network are presented in Table 5. Ranges of 100,150, and 200 kilometers are considered. The first two columns present the number of stations to be located and the drivers' tolerance for deviation. Columns (3) through (7) show results for vehicles with a range of 100 kilometers. In addition to the statistics provided in Table 3, column "Opt value, \%" shows the percentage of total vehicle flow covered. The following five columns correspond to solutions for vehicles of range 150 kilometers, and the rightmost five columns correspond to those for vehicles of range 200 kilometers. Even though our computer configuration is not the same as the one used in Yildiz et al. (2016), the two are comparable. According to PassMark Software (2017), our computer has a 1.147 times higher CPU mark rating. The results show that our $\mathrm{B} \& \mathrm{C}$ implementation outperforms the previous $\mathrm{B} \& \mathrm{P}$ algorithm: $\mathrm{B} \& \mathrm{C}-1$ is able to solve the instances even with $50 \%$ tolerance in less than two minutes, whereas the B\&P implementation could only handle $20 \%$ tolerance and terminated before reaching optimality after three hours for several instances.
Figure 3 plots the covered vehicle flow for different deviation tolerances when $p=1, \ldots, 35$ stations are optimally located and the vehicles have ranges of 100 , 150, and 200 kilometers in parts (a), (b), and (c), respectively. Coverage increases to $100 \%$ steadily in all three plots. The rate of increase is faster for higherdeviation tolerances. For instance, when range is 100 kilometers in Figure 3(a), in the $0 \%$ deviation curve, all demand can be covered when $p=30$. In the $50 \%$ deviation curve, by contrast, full coverage is reached when $p=15$. The rate of increase is also faster for higher vehicle ranges. When range increases to 200 kilometers in Figure 3(c), in the 0\% deviation curve, $100 \%$ coverage can now be reached by locating 20 stations rather than 30 stations as in the 100-kilometer scenario in Figure 3(a). Note that the impact of deviation tolerance on the covered flow is more pronounced when the range is shorter. For instance, when $p=5$ and range is 100 kilometers in Figure 3(a), coverage increases from $67.09 \%$ in $\hat{\lambda}=0 \%$ to $90.47 \%$ in $\hat{\lambda}=$ $50 \%$ (23.38\% difference). The increase is only $12.34 \%$ when the range is 200 kilometers in Figure 3(c) from 
Figure 5. Distribution of Vehicle Flow Grouped into Deviation Distance Intervals in the CA Network for Vehicles of 100-km Range and When $p=5$ and $\hat{\lambda}=50 \%$

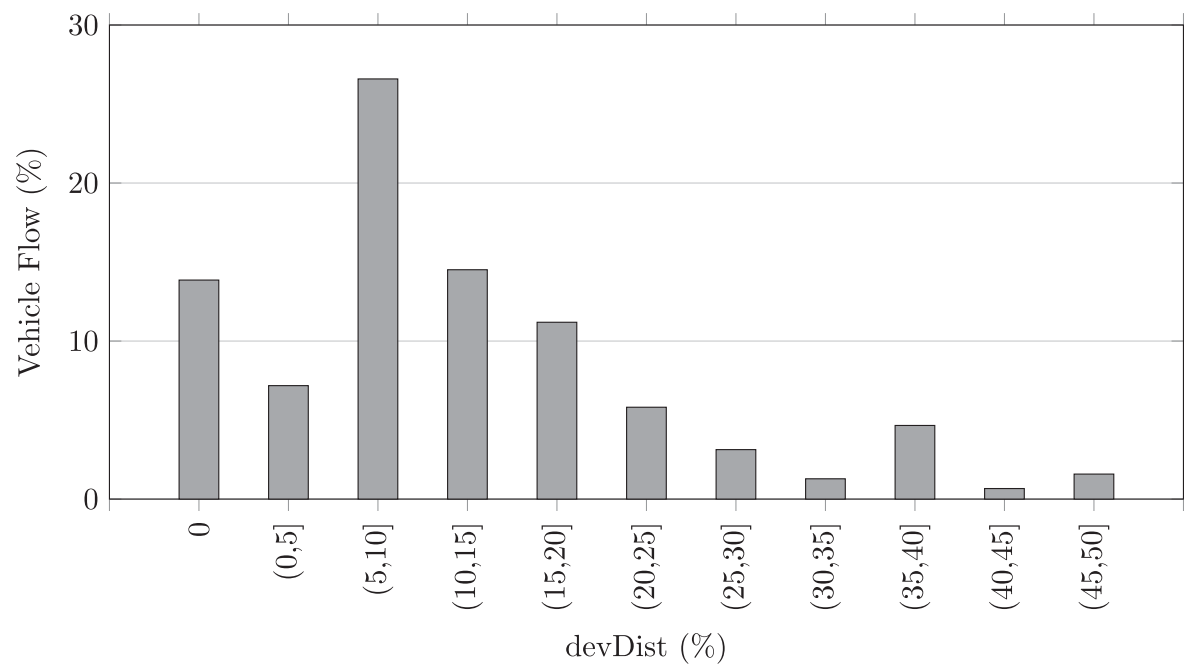

Note. The deviation distance on the horizontal axis is presented as a percentage of the shortest path.

$85.18 \%$ in $\hat{\lambda}=0 \%$ to $97.52 \%$ in $\hat{\lambda}=50 \%$. By locating only a single station (i.e., $p=1$ ) in Los Angeles in the southern part of California, $30.55 \%-43.85 \%$ of the vehicle flow can be covered, depending on the range of the vehicle and deviation tolerance of the drivers. Fifteen stations cover more than $90 \%$ of the demand for all range and tolerance levels considered in Figure 3. By locating 30 stations, all vehicle flows can be covered in all settings.

Note that when the problem is solved using our natural formulation, the optimal refueling station locations and the demand that can be covered are given by the $x$ and $y$ variables, respectively. Consider a demand $q$ and the corresponding OD pair $\left(o_{q}, d_{q}\right)$. We postprocess the optimal solution to determine the path that a vehicle flow takes by solving a shortest-path algorithm in the graph induced by the optimal station locations. Let $\delta_{o_{q} d_{q}}^{*}$ be the shortest path length in the induced graph. We then compare this length with $\delta_{o_{q} d_{q}}$, which is the shortest path length in the original road network. If $\delta_{O_{q} d_{q}}^{*}=\delta_{o_{q} d_{q}}$, then the vehicle flow travels on its shortest path. If $\delta_{o_{q} d_{q}}<\delta_{o_{q} d_{q}}^{*} \leq \lambda_{q}$, then the vehicle flow deviates from its shortest path to travel to its destination. If $\lambda_{q}<\delta_{o_{q} d_{q}}^{*}<\infty$, in other words, if the shortest path length is greater than the drivers' tolerance but finite, then the vehicle flow is not covered because there is no path of length at most $\lambda_{q}$. In this case, we refer to such flow as missed flow because of tolerance. If $\delta_{o_{q} d_{q}}^{*}=\infty$, that is, there is no path from the origin to the destination and these two nodes are disconnected, then the range is not long enough to travel between the located stations. Therefore, we refer to such flow as missed flow because of range.

Figure 4(a) shows the breakdown of covered and missed vehicle flows for different tolerances when five stations are optimally located in the CA network with vehicles of 100-kilometer range. This figure is representative to show the reasons for covering and missing vehicle flows. When the drivers are not tolerant to deviating (i.e., $0 \%$ tolerance), then $67.09 \%$ of the total vehicle flow can be covered. The coverage increases to $90.46 \%$ when the drivers tolerate $50 \%$ of their shortest paths. The deviating vehicle flow percentage increases for higher deviation tolerances, and vehicle flow traveling on their shortest paths decreases. We also observe in the figure that the main cause of missing vehicle flow is the limited range. The missed flow because of tolerance is at maximum $0.7 \%$ for $20 \%$ deviation tolerance in Figure 4(a). It is very rarely the case in all the scenarios that there exists a required infrastructure for vehicles to travel but the drivers are intolerant to deviating.

Figure 4(b) shows the covered and missed flow percentages in the optimal solutions when $p=1, \ldots, 35$ stations are located in the CA network with vehicles of 100-kilometer range and driver deviation tolerance of $10 \%$. The percentage of the deviating vehicle flow is increasing until full coverage is achieved at 20 stations. Higher numbers of stations being located in the network increases their availability on the shortest paths of the drivers, which leads to less vehicle flow deviating.

We now provide insights about the deviation distances. Similar to the deviation tolerance, we present the deviation distance as a percentage of the shortest path. We refer to the deviation distance as devDist, which is given by devDist $=100 \times\left(\delta_{o_{q} d_{q}}^{*}-\delta_{o_{q} d_{q}}\right) / \delta_{o_{q} d_{q}}$ for $q \in Q$. Figure 5 plots the distribution of vehicle flow grouped into devDist intervals in the CA network for vehicles of 100-kilometer range and when $p=5$ and $\hat{\lambda}=50 \%$. This figure is representative to show the 


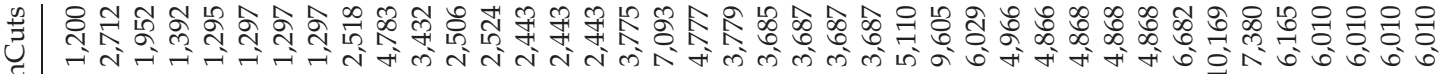

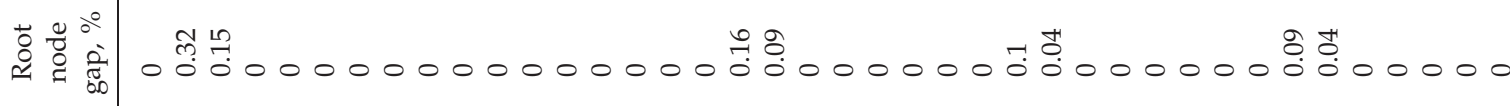

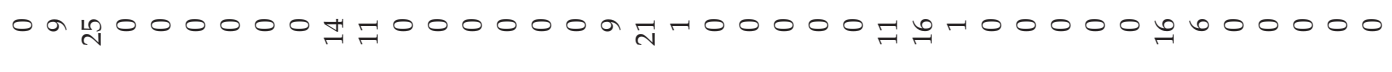

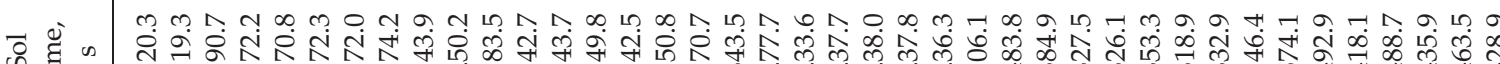

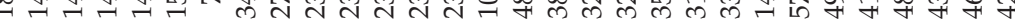

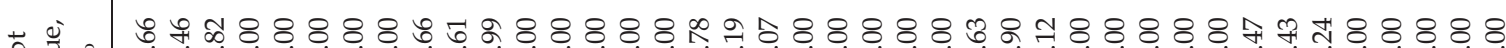
ईँ

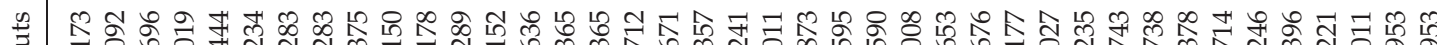

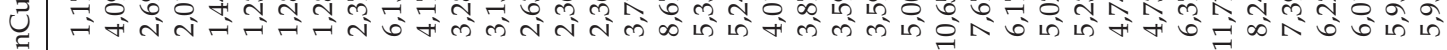

苟 向

范 б

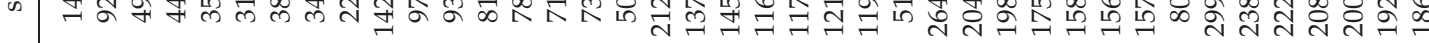

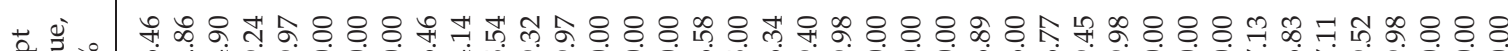

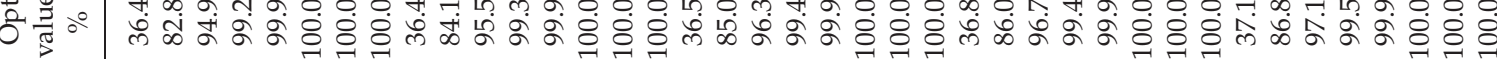

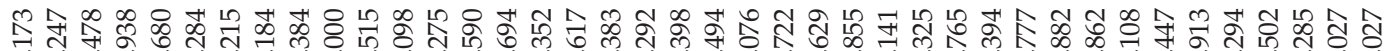

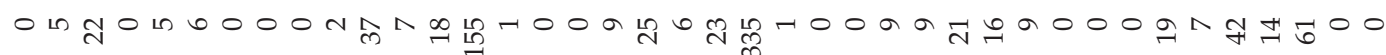

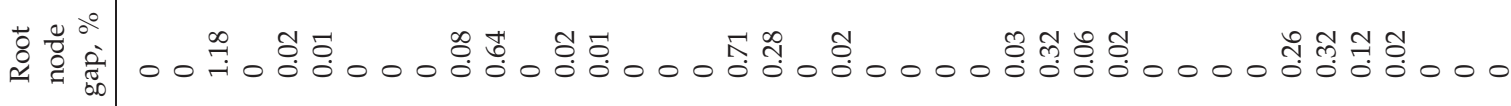

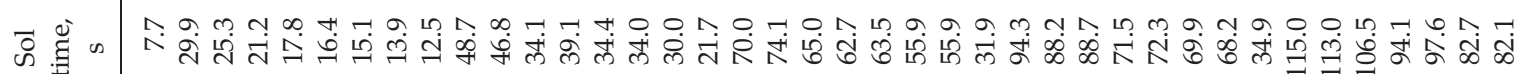

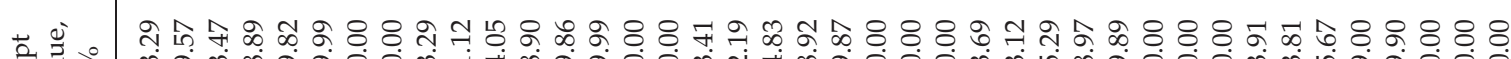

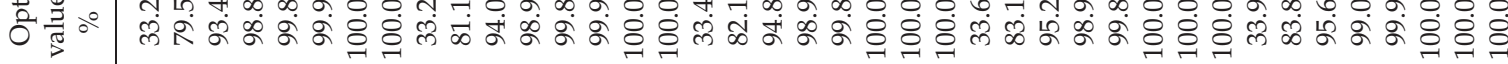

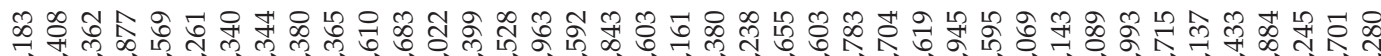

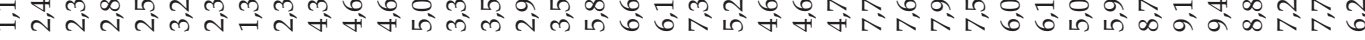

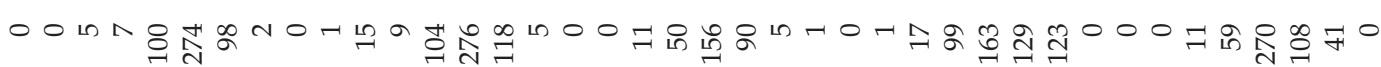

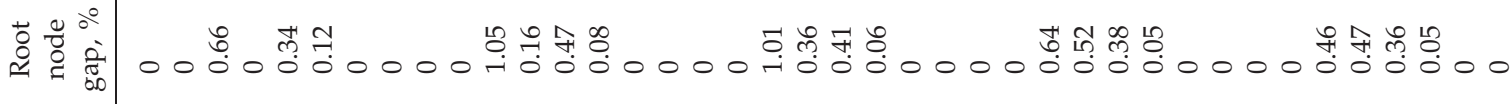

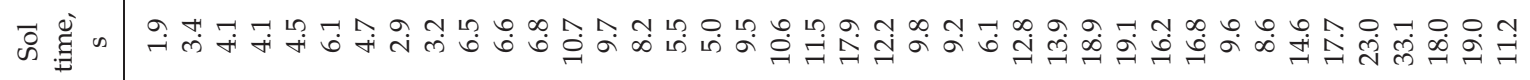

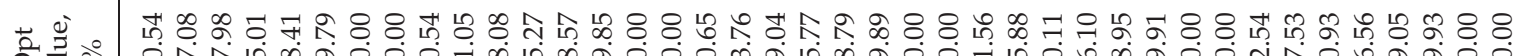

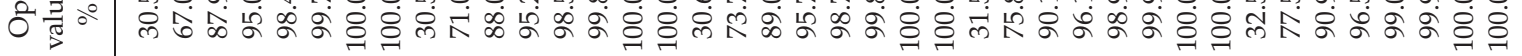




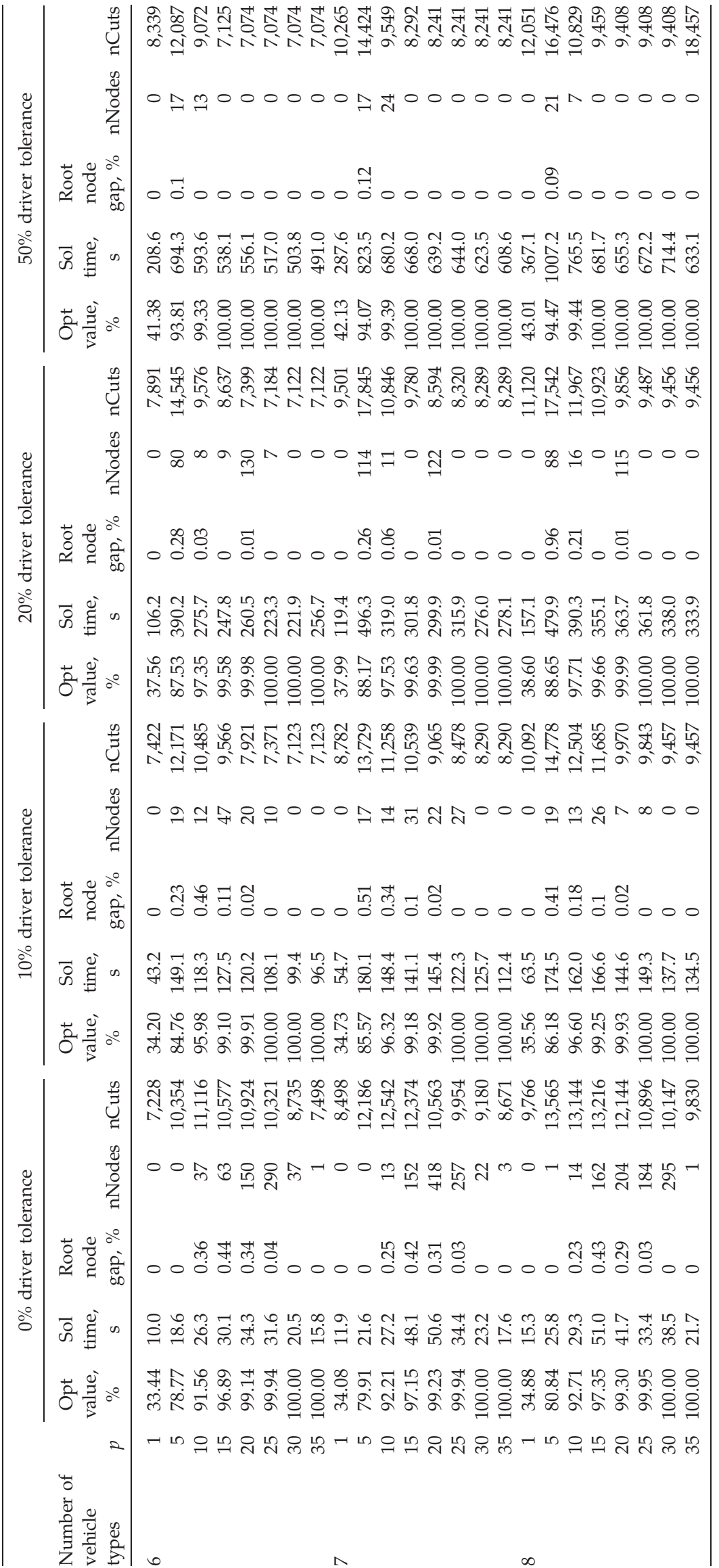


Figure 6. Solution Times for Different Number of Vehicle Types

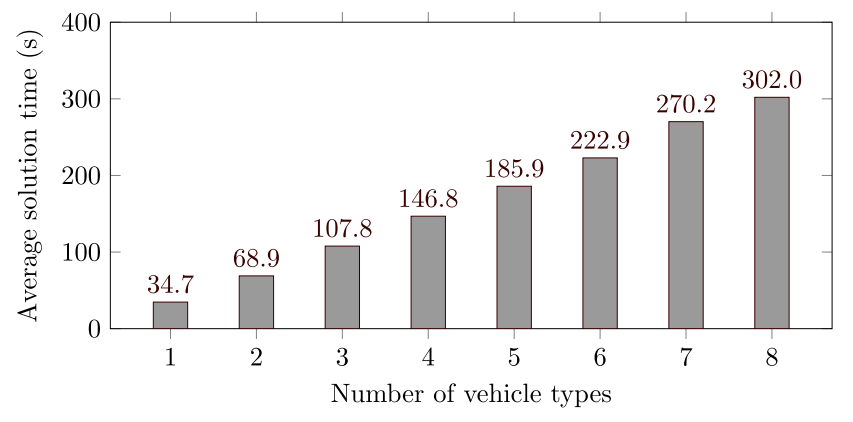

distribution of deviation distances. For the considered setting, Figure 4 (a) shows that $9.53 \%$ of the total vehicle flow is missed, $13.86 \%$ of the total vehicle flow drive on their shortest paths (shown in the first bar in Figure 5), and $76.61 \%$ of the total vehicle flow deviate (shown in bars 2-11 in Figure 5). The deviation peaks at $5 \%-10 \%$ deviation and gradually decreases until $50 \%$ tolerance level. The average devDist in the figure is $12.79 \%$. When all the instances in Table 5 are considered, the average devDist for $\hat{\lambda}=10 \%, \hat{\lambda}=20 \%$, and $\hat{\lambda}=50 \%$ are $3.1 \%, 5.8 \%$, and $10.6 \%$, respectively.

Observe that there are three critical parameters in the RSLP-R that affect the performance: (1) the deviation tolerance of the drivers, (2) the number of demands, and (3) the size of the network. In the CA network, we increased the deviation tolerance to $50 \%$ and observed that the model adapted to increasing deviations. In the following experiment, we concentrate on increasing the number of demands, and finally, we test our model against increasing network sizes in random graphs.

In the CA network, there are 1,167 OD pairs. The results presented in Table 5 are obtained assuming a single vehicle type traveling between each OD pair. In the following experiment, we add a new parameter: the number of vehicle types, and each OD pair is assigned the same number of vehicle types. Therefore, having eight vehicle types in an experiment refers to 9,336 distinct demands traveling between 1,167 OD pairs. The ranges of vehicles change between 100 and 275 kilometers by increments of 25 . In other words, when we have eight vehicle types running between the same OD pair, their ranges are 100, 125, .., 275 kilometers. In this regard, Table 6 presents results for the CA network for a different number of vehicle types. We again observe very small root node gaps. Figure 6 depicts the average solution times of the 32 instances corresponding to each vehicle type and shows that the solution times sublinearly increase by the number of vehicle types.

We also investigate the effect of increasing the number of OD pairs on solution times. For this purpose, we introduce two new sets of OD pairs. Recall that the
1,167 OD pair nodes in the previous experiments represent those urban centers with a population of 50,000 or more. This corresponds to approximately 5.71 million vehicles per year. In the first (and second, respectively) new set, we consider those urban centers with 30,000 (and 20,000, respectively) or more in population and 30 kilometers apart, corresponding to 1,874 (and 3,121, respectively) OD pairs and more than 5.91 million (and 6.11 million, respectively) vehicles per year. The results are reported in columns (3)-(20) of Table 7. All instances are solved in less than eight minutes. Apart from these two new OD pair sets, we also test unit vehicle flows between each OD pair. In our original experiments with 1,167 OD pairs, the vehicle flows are highly unbalanced, changing between 14.51 and 1,235,110 vehicles per year. For both balanced and unbalanced instances, the solution times and the root node gaps are consistently small, showing the strength of our solution methodology.

\subsection{Random Networks}

Random networks are generated to test the computational efficiency of the proposed B\&C-1 algorithm against increasing sizes of networks. For this purpose, four random graphs, details of which are presented in Table 2, are generated. For each network, 1,000, 2,000, and 4,000 random OD pairs are selected. For each graph and OD pair count, we test our algorithm for 10, $20,30,50$, and 100 stations. We conduct each experiment for $0 \%, 10 \%$, and $20 \%$ deviation tolerance. We consider a vehicle with a range of 100 kilometers. The results are presented in Table 8 . Three leftmost columns show the parameter settings. The "Best lbd, \%" column shows the best feasible solution at termination. "Opt Gap, \%" is the gap between the best upper and lower bounds at termination. "Sol time, s," "Root node gap, $\%$," "nNodes," and "nCuts" columns are as previously defined.

Observe that except for 17 instances, the algorithm was able to find the optimal solution within the onehour time limit. Average solution times are generally higher than those for the CA network. Note that even with large gaps at the root node, the algorithm still performs fairly fast in finding an optimal solution. According to our results, the algorithm performs well until the bottleneck is hit in instances with 1,000 nodes and 4,000 OD pairs.

\section{Conclusions}

In this study, we have proposed a natural formulation for the RSLP-R based on the notion of length-bounded node cuts and analyzed its polyhedral properties. We proposed a $B \& C$ algorithm as an exact solution method. For separating integer solutions, we devised a polynomial-time algorithm. For fractional solutions, we developed an integer programming model and 


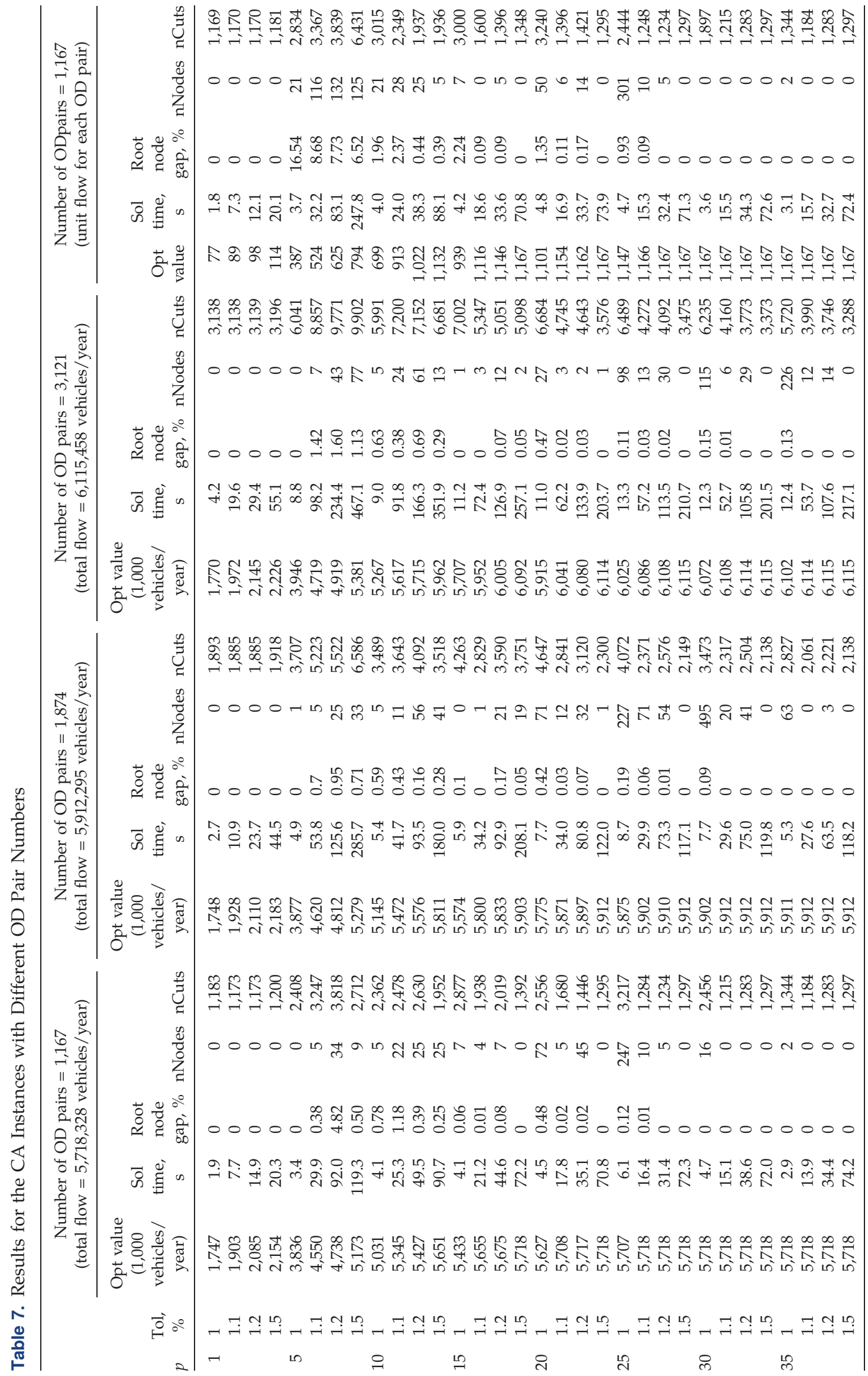


空 我

官

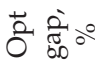

若迥

$\stackrel{0}{*}$

U

$\frac{0}{8}$

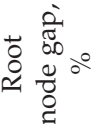

के

के 突。

㟧

贲

$\mathcal{J}$

总

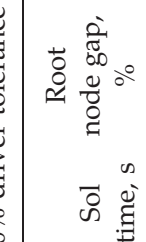

亏ेँ

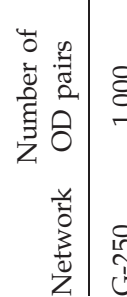

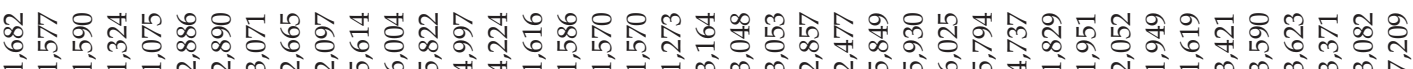

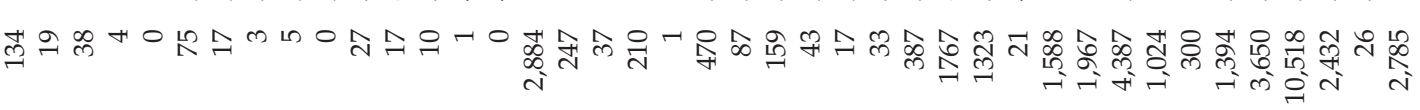

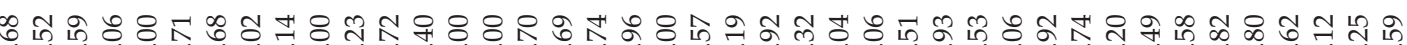

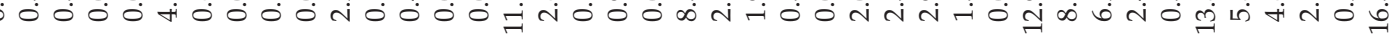

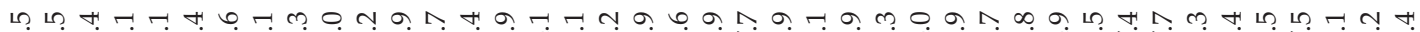

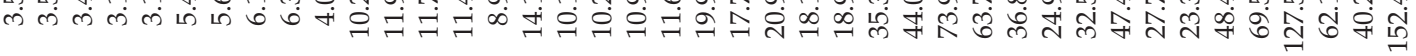

00000000000000000000000000000000000000000

邑 तें

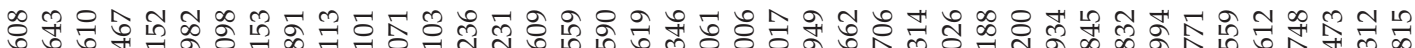
-

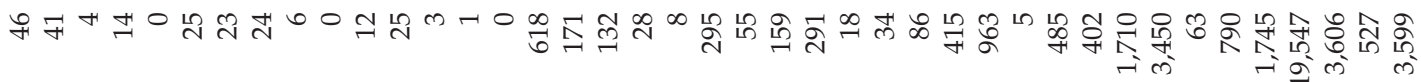

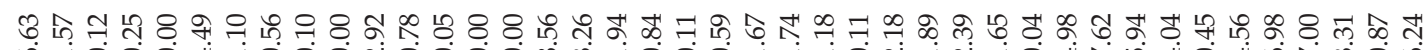

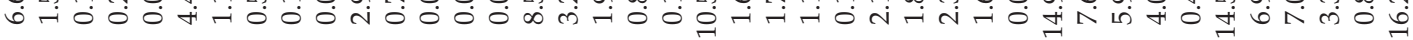

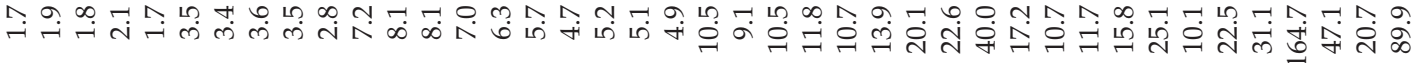
00000000000000000000000000000000000000000 늠유 \&

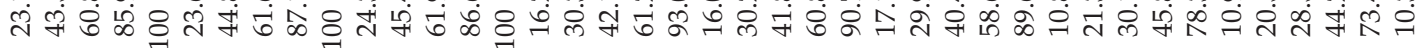

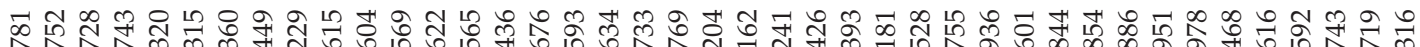

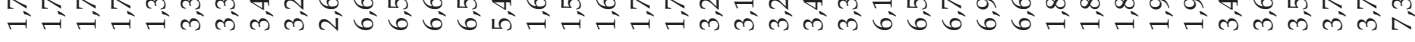

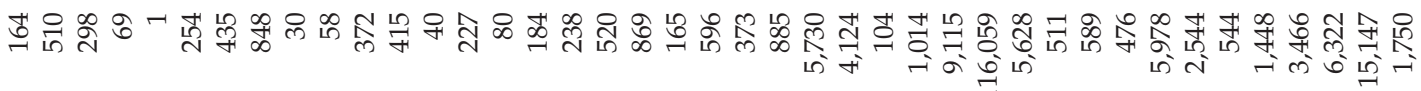

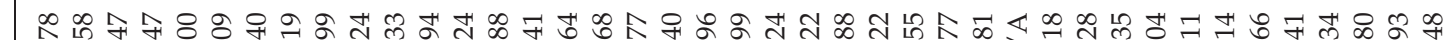

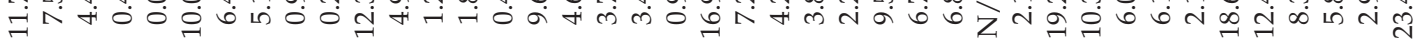

0 m

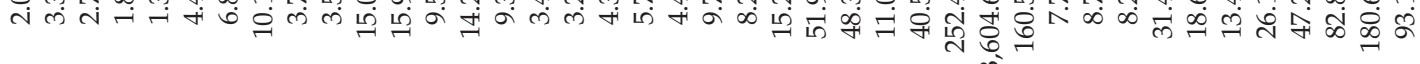

б

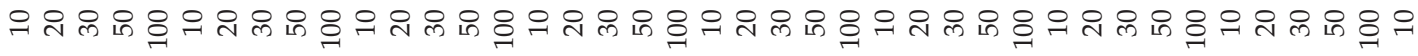




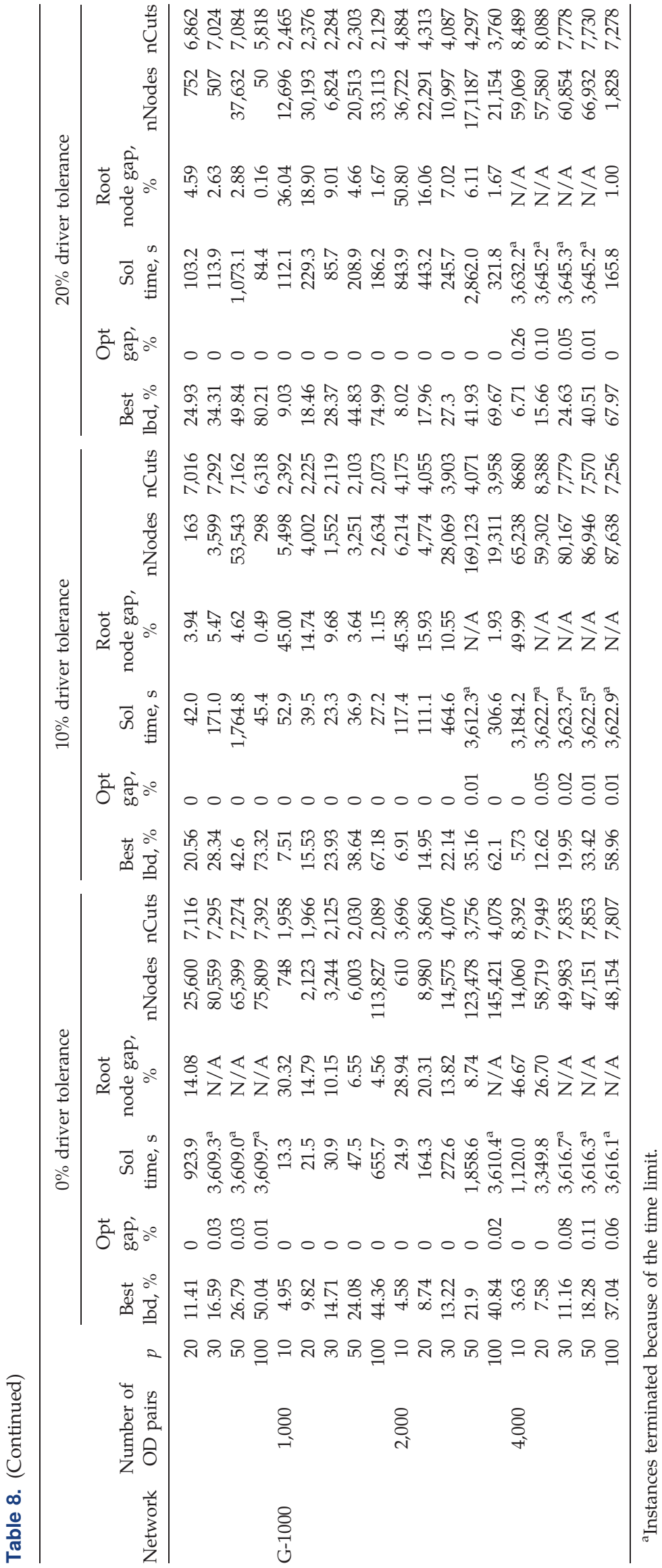


made use of a classical minimum cut algorithm as an efficient heuristic. Extensive computational studies showed that the solution times and the size of the solvable instances are improved significantly with respect to previously reported results by Yildız et al. (2016). We can address practically sized problem instances, which could only be solved previously by a B\&P algorithm in a three-hour time frame, in under two minutes. Our approach can also address multiple vehicle types and possible nonsimple path occurrences in the driver routes.

Our approach can easily be adapted to handle different driver tolerances. Rather than assuming a distance tolerance on the path, a bound on the number of refueling stops might be considered for the drivers. This special case of our problem can be solved by assuming unit length arcs in the transformed network and solving the RSLP-R on this transformed network.

For future research directions, capacities of the refueling stations can be accommodated into the problem, which would bring more realism. Considering uncertainties inherent in the problem, such as the vehicle flows between OD pairs, can also help in modeling the real world more closely.

\section{Acknowledgments}

The authors thank the associate editor and two referees for their constructive comments that helped improve the quality of this paper.

\section{References}

Arslan O, Karaşan OE (2016) A Benders decomposition approach for the charging station location problem with plug-in hybrid electric vehicles. Transportation Res. Part B: Methodological 93: 670-695.

Arslan O, Y1ldız B, Karaşan OE (2014) Impacts of battery characteristics, driver preferences and road network features on travel costs of a plug-in hybrid electric vehicle (PHEV) for longdistance trips. Energy Policy 74:168-178.

Baier G, Erlebach T, Hall A, Köhler E, Schilling H, Skutella M (2006) Length-bounded cuts and flows. Bugliesi M, Preneel B, Sassone V, Wegener I, eds. Internat. Colloquium Automata, Languages, Programming (Springer, Berlin, Heidelberg), 679-690.

Capar I, Kuby M, Leon VJ, Tsai YJ (2013) An arc-cover path-cover formulation and strategic analysis of alternative-fuel station locations. Eur. J. Oper. Res. 227(1):142-151.

Chen S, Ljubić I, Raghavan S (2010) The regenerator location problem. Networks 55(3):205-220.

Church R, ReVelle CS (1974) The maximal covering location problem. Papers Regional Sci. Assoc. 32(1):101-118.

Energy Information Administration (2017a) Alternative fuel vehicle data. Accessed January 17, 2017, http://www.eia.gov/renewable/ $\mathrm{afv} /$ users.php?fs=a.

Energy Information Administration (2017b) Annual energy outlook. Accessed May 18, 2014, http://www.eia.gov/outlooks/aeo/.
European Commission (2013) Clean power for transport. Accessed January 17, 2017, http://europa.eu/rapid/press-_release \_MEMO -_13-_24\_en.htm.

European Union (2009) Renewable energy directive. Accessed January 16, 2017, http://www.eur-_lex.europa.eu/legal-_content/ EN/TXT/PDF/?uri=CELEX:32009L0028.

Hodgson MJ (1990) A flow-capturing location-allocation model. Geographical Anal. 22(3):270-279.

IBM (2014) ILOG CPLEX optimization studio. Accessed February 13, 2017, http://www.cplex.com.

Kim JG, Kuby M (2012) The deviation-flow refueling location model for optimizing a network of refueling stations. Internat. J. Hydrogen Energy 37(6):5406-5420.

Kim JG, Kuby M (2013) A network transformation heuristic approach for the deviation flow refueling location model. Comput. Oper. Res. 40(4):1122-1131.

Kuby M, Lim S (2005) The flow-refueling location problem for alternative-fuel vehicles. Socio-Econom. Planning Sci. 39(2): 125-145.

Li X, Aneja Y (2017) Regenerator location problem: Polyhedral study and effective branch-and-cut algorithms. Eur. J. Oper. Res. 257(1): 25-40.

Lim S, Kuby M (2010) Heuristic algorithms for siting alternative-fuel stations using the flow-refueling location model. Eur. J. Oper. Res. 204(1):51-61.

Lovász L, Neumann-Lara V, Plummer M (1978) Mengerian theorems for paths of bounded length. Periodica Mathematica Hungarica 9(4):269-276.

Mahjoub AR, McCormick ST (2010) Max flow and min cut with bounded-length paths: Complexity, algorithms, and approximation. Math. Programming 124(1-2):271-284.

MirHassani SA, Ebrazi R (2013) A flexible reformulation of the refueling station location problem. Transportation Sci. 47(4): 617-628.

Sichi J, Kinable J, Michail D, Naveh B, Contributors (2017) JGraphTGraph algorithms and data structures in Java. Accessed February 13, 2017, http://www.jgrapht.org.

PassMark Software (2017) CPU performance comparison. Accessed May 18, 2017, https://www.cpubenchmark.net/compare.php ?cmp[]=2052 \\&cmp[]=1846.

Rahman Q, Bandyopadhyay S, Aneja Y (2015) Optimal regenerator placement in translucent optical networks. Optical Switching Networking 15:134-147.

Wang YW, Lin CC (2009) Locating road-vehicle refueling stations. Transportation Res. Part E: Logist. Transportation Rev. 45(5): 821-829.

Wang YW, Lin CC (2013) Locating multiple types of recharging stations for battery-powered electric vehicle transport. Transportation Res. Part E: Logist. Transportation Rev. 58:76-87.

Wang YW, Wang CR (2010) Locating passenger vehicle refueling stations. Transportation Res. Part E: Logist. Transportation Rev. 46(5):791-801.

Yetginer E, Karasan E (2003) Regenerator placement and traffic engineering with restoration in GMPLS networks. Photonic Network Comm. 6(2):139-149.

Yildız B, Karaşan OE (2015) Regenerator location problem and survivable extensions: A hub covering location perspective. Transportation Res. Part B: Methodological 71:32-55.

Yildız B, Arslan O, Karaşan OE (2016) A branch and price approach for routing and refueling station location model. Eur. J. Oper. Res. 248(3):815-826. 